\title{
Composição bromatológica e cinética da fermentação ruminal de rações contendo resíduos de babaçu ${ }^{1}$
}

\author{
Bromatological composition and kinetics of rumen fermentation for feeds containing \\ babassu residue
}

\author{
Luciano Fernandes Sousa ${ }^{2 *}$, Gilberto de Lima Macedo Júnior ${ }^{3}$, Rogério Pereira dos Santos ${ }^{4}$, André Guimarães \\ Maciel e Silva 5 e Iran Borges ${ }^{6}$
}

\begin{abstract}
RESUMO - Objetivou-se com o experimento demonstrar a composição bromatológica e avaliar a cinética de fermentação e degradabilidade ruminal efetiva in vitro, por meio da técnica "Hohenheim Gas Test", da farinha do mesocarpo do babaçu I ${ }^{\circledR}$ e II ${ }^{\circledR}$ (FMBI e FMBII) e de rações com diferentes níveis de inclusão. Foram conduzidos dois ensaios: um com os subprodutos puros e milho e outro com rações com inclusões da FMBI e FMBII nas seguintes proporções: 0; 7,5; 15,0 e 22,5\% da matéria seca total. O delineamento foi o de blocos casualizados sendo seis blocos no primeiro e cinco no segundo ensaio. Os tempos de mensuração dos gases produzidos foram 3; 6; 9; 12; 24; 48; 72 e 96 horas. As farinhas FMBI e FMBII apresentaram composições bromatológicas diferentes, em relação aos constituintes da fibra (FDN, FDA e Lignina). As farinhas FMBI e FMBII apresentaram padrões de cinética de fermentação inferiores aos padrões de fermentação do milho grão. Com o aumento da proporção de FMBI e FMBII na ração, reduz-se a produção de gases por tempo de incubação. Em virtude do exposto, pode-se afirmar que as farinhas do mesocarpo I e II, apesar de serem fontes de amido, não apresentaram fermentação ruminal semelhante à fonte amilácea padrão, o grão de milho.
\end{abstract}

Palavras-chave: Alimentação. Orbignya martiana. Produção de gases. Ruminantes. Subprodutos.

\begin{abstract}
The objective of the experiment was to demonstrate the bromatological composition, and evaluate the fermentation kinetics and effective ruminal degradability in vitro, using the "Hohenheim Gas Test" technique, of babassu mesocarp flour $\mathrm{I}^{\circledR}$ and $\mathrm{II}^{\circledR}$ (FMBI and FMBII) and of feeds at different levels of inclusion. Two trials were carried out: one of pure byproducts and corn, and another of feeds with inclusions of FMBI and FMBII in the following proportions: $0,7.5,15.0$ and $22.5 \%$ of the total dry matter. The experimental design was of randomized blocks, with six blocks in the first trial and five in the second. Measurement times for the gases produced were 3, 6, 9, 12, 24, 48, 72 and 96 hours. The FMBI and FMBII flours showed different bromatological composition for the fibre components (FDN, FDA and Lignin). The FMBI and FMBII flours also showed patterns for fermentation kinetics inferior to the fermentation patterns of corn grain. Increasing the ratio of FMBI and FMBII in the feed reduces gas production for time of incubation. In view of the above, it can be said that mesocarp flours I and II, despite being sources of starch, did not present ruminal fermentation similar to corn grain, the standard source of starch.
\end{abstract}

Key words: Feeding. Orbignya martiana. Production of gases. Ruminants. By-products.

\footnotetext{
*Autor para correspondência

${ }^{1}$ Recebido para publicação em 04/04/2012; aprovado em 14/09/2013

Pesquisa desenvolvida como parte do plano de trabalho de estágio probatório do primeiro autor financiada pelo CNPq

${ }^{2}$ Programa de Pós-Graduação em Ciência Animal Tropical, Curso de Zootecnia, Universidade Federal do Tocantins/UFT, Araguaína-TO, Brasil, luciano.sousa@uft.edu.br

${ }^{3}$ Programa de Pós-Graduação em Ciência Animal Tropical/UFT, Uberlândia-MG, Brasil, gilbertomacedojr@gmail.com

${ }^{4}$ Programa de Pós-Graduação em Ciência Animal Tropical/UFT, Araguaína-TO, Brasil, roger.rps @ hotmail.com

${ }_{5}^{5}$ Programa de Pós-Graduação em Ciência Animal, Instituto de Medicina Veterinária, Universidade Federal do Pará/NCDAR/UFPA, Castanhal-PA, Brasil, andregms@gmail.com

${ }^{6}$ Programa de Pós-Graduação em Zootecnia, Departamento de Zootecnia da Escola de Veterinária, Universidade Federal de Minas Gerais/UFMG, Belo Horizonte-MG, Brasil, iran@ vet.ufmg.br
} 


\section{INTRODUÇÃO}

A busca por alternativas alimentares para ruminantes é um esforço constante na nutrição animal, pois a alimentação representa cerca de 60 a $70 \%$ do custo total da produção de qualquer espécie de interesse zootécnico (ABDALLA et al., 2008; NEIVA JÚNIOR et al., 2007). Visando minimizar os custos da ração, subprodutos industriais têm sido utilizados, como fontes alternativas de alimentos de menor custo, em substituição a produtos como o milho e a soja.

Nas regiões Norte e Nordeste a palmeira do babaçu existe em abundância e seus frutos são utilizados por muitos agricultores como fonte de renda na forma de artesanato, carvão e também na nutrição animal (SOLER et al., 2007).

O babaçu é uma palmeira alta e copada do gênero Orbignia (Orbignya sp.), que se divide em duas espécies: Orbignya oleifera (babaçu do cerrado) e Orbignya martiana (babaçu da floresta). Pode atingir 10 a 20 metros de altura. Cada palmeira pode produzir 2.000 cocos por ano, de junho a janeiro, os quais têm seis a $13 \mathrm{~cm}$ de comprimento (EMPRESA BRASILEIRA DE PESQUISA AGROPECUÁRIA, 1984).

As farinhas do mesocarpo do babaçu (FMB) $\mathrm{I}^{\circledR}$ e II ${ }^{\circledR}$ (FMBI e FMBII) são extraídas dos frutos dessa palmácea nativa da América do Sul, os quais consistem em um dos principais produtos do extrativismo vegetal no Norte do Estado do Tocantins.

A composição física do fruto indica quatro partes aproveitáveis: epicarpo (11\%), mesocarpo (23\%), endocarpo (59\%) e amêndoa (7\%). A casca (93\%), conjunto formado pelo epicarpo, mesocarpo e endocarpo, é normalmente desprezada nos processos artesanais de quebra manual e extração das amêndoas, já na indústria seu aproveitamento se dá de forma integral o que gera uma série de subprodutos (EMPRESA BRASILEIRA DE PESQUISA AGROPECUÁRIA, 1984).

As FMBI e FMBII são oriundas do processo de pelagem do coco, que consiste na retirada do mesocarpo e epicarpo pela extração mecânica na industrialização do coco de babaçu, para posteriormente proceder a sua serragem ao meio para extração das amêndoas na industrialização dos cocos (Figura 1). Após a extração do mesocarpo e epicarpo separa-se o epicarpo do mesocarpo mecanicamente por meio de peneiras com furos de diâmetros diferentes e é neste peneiramento que se dá a distinção das duas farinhas. O mesocarpo do fruto do babaçu, devido à sua composição química e ao seu teor de amido, que varia de 63,75 a $71,29 \%$ (EMPRESA BRASILEIRA DE PESQUISA AGROPECUÁRIA, 1984), vem sendo utilizado como componente de rações para animais, entretanto há contaminação no momento de extração do mesocarpo pelo epicarpo e endocarpo, que são fibrosos, pode tornar a FMB um subproduto com maior teor de fibra.

Apesar de já ser utilizada como alimento alternativo, dados de fermentação ruminal deste subproduto ainda não foram publicados, justificando a realização deste trabalho, uma vez que informações sobre a cinética de fermentação são importantes ferramentas na utilização de subprodutos em programas de nutrição e alimentação de ruminantes (MERTENS, 1997).

Dentre as metodologias utilizadas para obtenção de parâmetros da cinética de fermentação ruminal pode-se destacar as técnicas de produção de gases em suas diversas variações, as quais segundo Azarfar et al. (2009) são rápidas, precisas e em algumas modalidades metodológicas, de custo reduzido, o que as tornam atrativas para centros de investigação que

Figura 1 - Coco de babaçu e suas partes

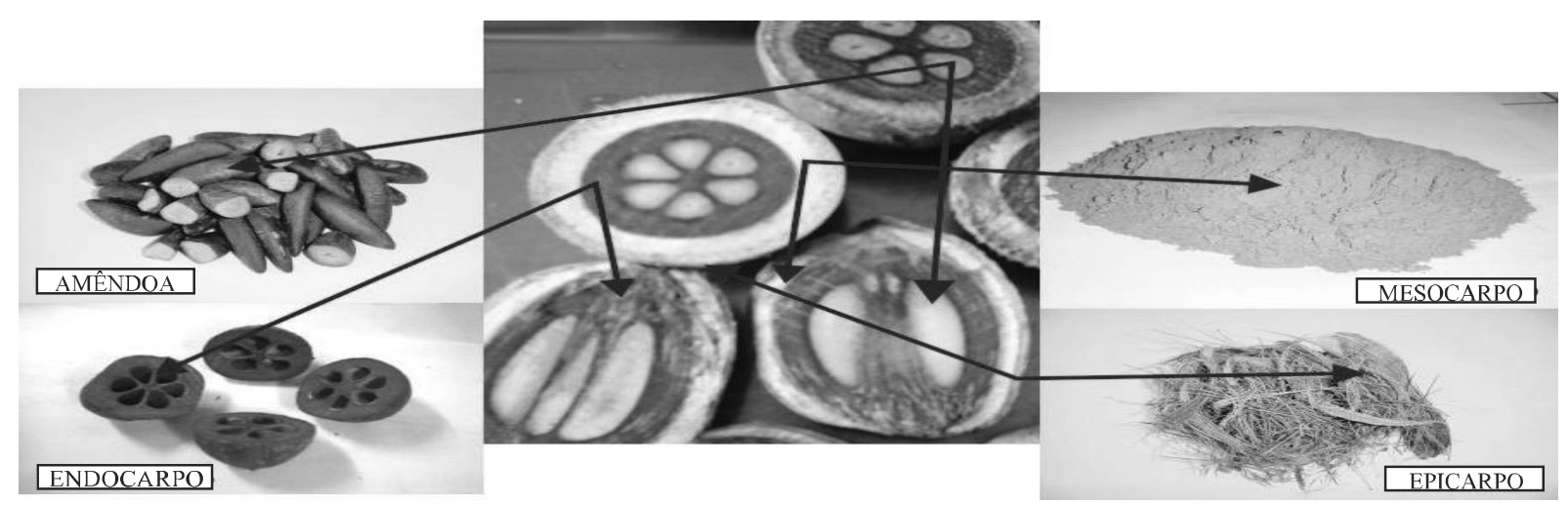

Fonte: Tobasa Bioindustrial de Babaçu S.A.(2011) 
trabalham com avaliação de alimentos alternativos para ruminantes.

São vantagens desta técnica ainda sua simplicidade de uso e a possibilidade de processar grande número de amostras em curto espaço de tempo (RYMER et al., 2005; SALLAM et al., 2007). Entretanto, algumas desvantagens são atribuídas a estas técnicas, entre elas estão o fato de geralmente usarem amostras reduzidas dificultando a homogeneização da amostra estudada e a dificuldade de padronização dos inóculos dos diferentes estudos o que dificulta maiores extrapolações (KRISHNAMOORTHY et al., 2005).

Objetivou-se com este trabalho demonstrar a composição bromatológica e avaliar a cinética de fermentação ruminal e a degradabilidade efetiva das farinhas do mesocarpo do babaçu $\mathrm{I}^{\circledR}$ e $\mathrm{II}^{\circledR}$ oriundas dos processos de industrialização do babaçu e uma fonte amilácea padrão (milho em grão) e de rações com inclusões desses alimentos.

\section{MATERIAL E MÉTODOS}

O experimento foi conduzido no Setor de Ensaios de Produção de Gases do Laboratório de Nutrição Animal da Escola de Medicina Veterinária e Zootecnia (EMVZ) da Universidade Federal do Tocantins (UFT).

As farinhas do mesocarpo do babaçu $\mathrm{I}^{\circledR}$ e $\mathrm{II}^{\circledR}$, foram adquiridas da indústria TOBASA-S.A. e juntamente com o milho em grão, foram levadas ao Laboratório de Nutrição Animal da EMVZ, onde foram moídas em moinho tipo "Willey", em peneiras de $1 \mathrm{~mm}$ e posteriormente encaminhadas ao Laboratório de Nutrição Animal da Escola de Veterinária da Universidade Federal de Minas Gerais (UFMG), onde foram analisadas para obtenção dos teores de matéria seca (MS), matéria mineral $(\mathrm{MM})$, proteína bruta (PB), fibra em detergente neutro (FDN), fibra em detergente ácido (FDA), hemicelulose (HCEL), lignina (LIG), celulose (CEL) com o aparelho “Ankon ${ }^{\circledR ”}$ pelo método sequencial, além do nitrogênio insolúvel em detergente neutro (NIDN) e nitrogênio insolúvel em detergente ácido (NIDA) (ASSOCIATION OF OFFICIAL ANALYTICAL CHEMISTS, 2002).

As rações com diferentes níveis de inclusão FMBI e FMBII $(0 ; 7,5 ; 15$ e $22,5 \%$ da MS total da ração) foram formuladas para ser isonitrogenadas, isoenergéticas e com teores de FDN semelhantes (Tabela 1).

$\mathrm{O}$ delineamento experimental foi blocos casualizados com três tratamentos (milho, FMBI e FBMII) para os ensaios com alimentos puros e quatro

Tabela 1 - Rações experimentais obtidas com inclusões das farinhas do mesocarpo do babaçu ${ }^{\circledR}$ e II ${ }^{\circledR}$ (FMBI e FMBII)

\begin{tabular}{|c|c|c|c|c|}
\hline \multirow{2}{*}{ Ingredientes } & \multicolumn{4}{|c|}{ Níveis de inclusão da farinha do mesocarpo do babaçu I® (\% da MS) } \\
\hline & 0,00 & 7,50 & 15,00 & 22,50 \\
\hline Silagem de Napier & 53,34 & 47,13 & 40,96 & 34,78 \\
\hline FBMI & 0,00 & 7,50 & 15,00 & 22,50 \\
\hline Milho & 25,63 & 24,32 & 22,98 & 21,66 \\
\hline Farelo de soja & 16,03 & 15,91 & 15,79 & 15,69 \\
\hline Fosfato & 0,54 & 0,69 & 0,84 & 0,96 \\
\hline Sal mineral & 3,00 & 3,00 & 3,00 & 3,00 \\
\hline Calcário dolomítico & 1,46 & 1,45 & 1,43 & 1,41 \\
\hline \multirow{2}{*}{ Ingredientes } & \multicolumn{4}{|c|}{ Níveis de inclusão da farinha do mesocarpo do babaçu II® (\% da MS) } \\
\hline & 0,00 & 7,50 & 15,00 & 22,50 \\
\hline Silagem de Napier & 53,31 & 48,21 & 43,10 & 38,00 \\
\hline FBMII & 0,00 & 7,50 & 15,00 & 22,50 \\
\hline Milho & 25,63 & 22,79 & 19,96 & 17,12 \\
\hline Farelo de soja & 16,06 & 16,39 & 16,75 & 17,11 \\
\hline Fosfato & 0,54 & 0,67 & 0,77 & 0,88 \\
\hline Inerte & 3,00 & 3,00 & 3,00 & 3,00 \\
\hline Calcário dolomítico & 1,46 & 1,44 & 1,42 & 1,39 \\
\hline
\end{tabular}


para os ensaios com as rações com diferentes inclusões e cinco blocos (inóculos de diferentes vacas).

Tais alimentos e rações foram submetidas a um ensaio de produção de gases e degradabilidade por meio de uma adaptação da técnica "Hohenheim Gas Test" desenvolvida por Menke et al. (1979), utilizando seringas de vidro de $100 \mathrm{ml}$ graduadas para mensuração da produção de gases. Foram usados 0,2 gramas dos alimentos e das rações testadas, incubando estes nas seringas com $10 \mathrm{ml}$ de inóculo e $20 \mathrm{ml}$ de meio de cultura.

O líquido ruminal (inóculo) foi coletado, antes do fornecimento da alimentação matinal de cinco bovinos fistulados no rúmen, recebendo rações formuladas para atender as exigências de mantença.

Os seguintes horários foram usados para medida do volume dos gases produzidos: $3 ; 6 ; 9 ; 12 ; 24 ; 48 ; 72$ e 96 horas após inoculação. Os dados obtidos foram corridos utilizando brancos (seringas sem amostra) e os valores ajustados para produção por grama de MS. Após tal procedimento, o modelo de France et al. (1993) foi ajustado aos dados, como expresso na equação 1 :

$Y=A \cdot\left\{1-\exp ^{\left[-b \cdot(t \cdot T)-c \cdot\left(V_{t}-\sqrt{ } T\right)\right]}\right\}$

Foram obtidos os seguintes parâmetros referentes à cinética de produção de gases: produção de gases acumulada " $Y$ " em mL, tempo de incubação " $t$ " em horas, total de gases " $A$ " em $\mathrm{mL}$, tempo de colonização " $T$ " em horas e taxa de degradação fracional " $\mu$ " em $\mathrm{h}^{-1}$. As equações geradas foram comparadas por meio de teste de paralelismo e identidade de curvas de acordo com Regazzi e Silva (2004) ( $<$ < 0,05), ao nível de 5\% probabilidade de erro. Foram estimadas também as degradabilidades efetivas, como expresso abaixo:

$D E=S_{\mathrm{o}} \cdot \exp ^{-k t} \cdot(1-k I) /\left(S_{\mathrm{o}}+U_{\mathrm{o}}\right)$

Em que: $D E=$ degradabilidade efetiva, $k=$ taxa de passagem; sendo calculado para $\mathrm{k}=0,05 ; 0,06 ; 0,07$ e 0,08 e S0 e U0 = frações inicialmente fermentáveis e frações não fermentáveis, respectivamente, sendo:

$I=\exp ^{\left[-b \cdot(t \cdot T)+c \cdot\left({ }^{t}-\sqrt{ } T\right)\right]} d t$

\section{RESULTADO E DISCUSSÃO}

Pode-se verificar que as duas farinhas apresentam baixos teores de proteína bruta, quando comparadas ao milho (Tabela 2). Esse dado pode limitar o uso desses alimentos em virtude de afetar a digestibilidade dos constituintes fibrosos para diferentes espécies zootécnicas de ruminantes (VAN SOEST, 1994). Observa-se também que a quantidade de extrato etéreo é baixa nas duas farinhas em comparação ao MG.

A quantidade de fibra em detergente neutro e ácido (FDN e FDA) varia bastante entre as farinhas FMBI e FMBII e estes teores são bem superiores aos teores de FDN e FDA do milho (Tabela 2). A FMBII apresenta menor quantidade de FDN, que a FMBI e verifica-se também que a FMBII apresenta menor quantidade de lignina, constituinte indigestível para os ruminantes. Essa diferença é superior a 57\%. Assim, pode-se inferir que a FMBII poderá ter melhor aproveitamento pelos animais em determinada inclusão na ração.

Os valores de degradabilidade efetiva da MS (DEMS), potencial máximo de produção de gás (A), tempo de colonização (T) e taxa de produção de gás $(\mu)$, das FMBI, FMBII e MG estão demonstrados na Tabela 3.

Os maiores valores de A, $\mu$ e DEMS e o menor valor de T obtido com o milho grão podem estar relacionados aos menores teores de FDN e lignina, em relação às farinhas do mesocarpo do babaçu, já que esses constituintes dos alimentos interferem nos processos de colonização e fermentação dos alimentos pela microbiota presente no rúmen (VAN SOEST, 1994). Santos et al. (2003) testaram a fermentação de rações com diferentes alimentos e observaram maior produção de gases em rações com fubá de milho, devido a este alimento ser rico em carboidratos prontamente fermentáveis, como o amido, propiciando maior fermentação ruminal, resultando em maior produção de gases, quando comparado com alimento com maior proporção de carboidratos estruturais (parede celular).

Segundo Guimarães Júnior et al. (2008), o tempo de colonização corresponde ao tempo compreendido entre o início da incubação até a ação microbiana, sendo influenciado pela ração testada, como maior presença de substratos prontamente fermentáveis, e por características físicas e químicas da parede celular da amostra. As FMBI e FMBII testadas apresentam fração fibrosa muito lignificada (Tabela 2) o que dificulta a colonização de fermentação da FDN destes alimentos, pois a lignina é indigestível em ambiente ruminal, sendo tóxica para muitos microorganismos ruminais (VAN SOEST, 1994).

Na Tabela 4 estão apresentadas as equações e as curvas advindas da análise de regressão dos dados de cinética de fermentação ruminal das FMBI, FMBII e MG. A equação do MG apresentou-se distinta $(\mathrm{P}<0,05)$ das equações dos demais alimentos (FMBI e FMBII), resultado este que está provavelmente relacionado às diferenças bromatológicas das FMBI e FMBII e do MG demonstradas na Tabela 2. 
Tabela 2 - Composição bromatológica percentual das farinhas do mesocarpo do babaçu I ${ }^{\circledR}$ e II $^{\circledR}($ FMBI e FMBII) e do milho grão (MG)

\begin{tabular}{lccr}
\hline \multirow{2}{*}{\multicolumn{1}{c}{ Parâmetros bromatológicos }} & \multicolumn{3}{c}{ Tratamentos } \\
\cline { 2 - 4 } & FMBI $^{1}$ & FMBII $^{1}$ & MG $^{2}$ \\
\hline Matéria seca (MS) (\%) & 87,83 & 87,41 & 87,54 \\
Matéria mineral (\% da MS) & 4,36 & 3,87 & 1,50 \\
Proteína bruta (\% da MS) & 6,09 & 4,44 & 9,15 \\
Extrato etéreo (\% da MS) & 1,29 & 0,5 & 3,97 \\
Fibra em detergente neutro (\% da MS) & 60,2 & 44,28 & 15,28 \\
Fibra em detergente ácido (\% da MS) & 44,02 & 32,75 & 3,78 \\
Hemicelulose (\% da MS) & 16,17 & 11,53 & 11,50 \\
Celulose (\% da MS) & 26,07 & 22,02 & 3,51 \\
Lignina (\% da MS) & 21,16 & 12,11 & 1,66 \\
Nitrogênio (N) insolúvel em detergente neutro (\% da N total) & 0,25 & 0,21 & 0,11 \\
Nitrogênio (N) insolúvel em detergente ácido (\% da N total) & 0,31 & 0,27 & 0,09 \\
\hline
\end{tabular}

${ }^{1}$ Farinhas do mesocarpo do babaçu I ${ }^{\circledR}$ e II ${ }^{\circledR}$ (FMB I, FMB II); ${ }^{2}$ Milho grão (MG)

Tabela 3 - Parâmetros da cinética de fermentação ruminal in vitro das farinhas do mesocarpo do babaçu I ${ }^{\circledR}$ e II ${ }^{\circledR}$ (FMBI e FMBII) e do milho grão (MG), utilizando o modelo de France e degradabilidade efetiva

\begin{tabular}{lccc}
\hline \multirow{2}{*}{ Parâmetros de France } & \multicolumn{3}{c}{ Tratamentos } \\
\cline { 2 - 4 } & FMBI $^{1}$ & FMBII $^{1}$ & MG $^{2}$ \\
\hline $\mathrm{A}^{*}(\mathrm{~mL})$ & 204,91 & 219,39 & 284,46 \\
$\mathrm{~T}^{*}$ (horas:minutos) & $5: 45$ & $5: 40$ & $0: 04$ \\
$\mu^{*}$ & 0,0454 & 0,0454 & 0,0570 \\
DEMS** (5\%) (\%) & 34,01 & 39,48 & 66,47 \\
DEMS (6\%) (\%) & 31,78 & 36,94 & 65,77 \\
DEMS (7\%) (\%) & 29,71 & 34,56 & 65,06 \\
DEMS (8\%) (\%) & 27,76 & 32,32 & 64,36 \\
\hline
\end{tabular}

A - Total de gases $(\mathrm{mL}) ; \mathrm{T}$ - Tempo de colonização (horas); $\mu$ - Taxa de degradação fracional $\left(\mathrm{h}^{-1}\right)$; * Parâmetros estimados pelo modelo de France et al. (1993); **Degradabilidade efetiva da matéria seca; ${ }^{1}$ Farinhas amiláceas $I^{\circledast}$ e II ${ }^{\circledast}$ (FMBI e FMBII); Milho grão (MG)

Tabela 4 - Equações da produção acumulativa de gases (PCG), em mL.g-1 de MS das farinhas do mesocarpo do babaçu I ${ }^{\circledR}$ e II ${ }^{\circledR}($ FMBI e FMBII) e do milho grão (MG)

\begin{tabular}{lcc}
\hline \multicolumn{1}{c}{ Alimentos } & Equações (Modelo de France) & $\mathrm{R}^{2}(\%)$ \\
\hline FMBI $^{1}$ & $\mathrm{Y}=204,9175 \times\left\{1-\exp ^{[-(0,0639) \times(\mathrm{t}-5,7663)-(-0,1661) \times(\sqrt{ } \mathrm{t}-\sqrt{ } 5,7663)}\right\}$ a B & 88,20 \\
FMBII $^{1}$ & $\mathrm{Y}=219,3914 \times\left\{1-\exp ^{[-(0,0637) \times(\mathrm{t}-5,6710)-(-0,1631) \times(\sqrt{ } \mathrm{t}-\sqrt{5} 5,6710)]}\right\}$ a B & 83,20 \\
$\mathrm{MG}^{2}$ & $\mathrm{Y}=284,4572 \times\left\{1-\exp ^{[-(0,0700) \times(\mathrm{t}-0,0805)-(-0,1156) \times(\sqrt{ } \mathrm{t}-\sqrt{ } 0,0805)]}\right\}$ a A & 91,26 \\
\hline
\end{tabular}

Equações acompanhadas por letras minúsculas, iguais na mesma coluna, são paralelas pelo teste de paralelismo de curvas a 5\% de probabilidade. Equações acompanhadas por letras maiúsculas, iguais na mesma coluna, são idênticas pelo teste de identidade de curvas a 5\% (REGAZZI e

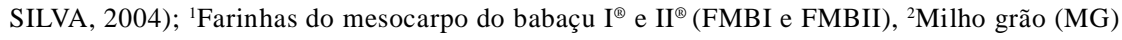


Os menores valores da degradabilidade da MS nas farinhas de mesocarpo do babaçu devido à fração da fibra não digestível, principalmente à lignina, em quantidade elevada em relação ao milho grão, influenciam negativamente a degradabilidade, ocasionando menor produção de gases.

Os teores de FDN dos alimentos influenciaram a cinética de fermentação ruminal. A composição bromatológica e a cinética de produção de gases obtidas neste ensaio evidenciam possível restrição no uso desses alimentos em rações para ruminantes. Principalmente em animais de alta exigência nutricional, como ruminantes de alto potencial produtivo, no terço final de gestação ou no pico de lactação.

A inclusão de níveis crescentes da FMBI no concentrado ocasionou proporcionalmente elevação nos valores de lignina (Tabela 5). O teor e o tipo da FDN e FDA são importantes parâmetros para avaliar a qualidade de um alimento, porque sua fermentação varia extensamente no rúmen, podendo influenciar o desempenho do animal (MERTENS, 1997).

A fração de FDN está relacionada principalmente à redução do consumo de alimento, enquanto que a FDA e a lignina estão diretamente relacionadas à digestibilidade do alimento. A adição da FMBI às rações aumentou os níveis de FDA, indicando baixa degradabilidade desse alimento. Tais resultados corroboram com observações de Mendes et al. (2005) que relataram que o fator principal de limitação da digestibilidade da matéria seca em volumosos ricos em fibra (farelo de girassol) é o FDA, sendo a lignina o componente desta FDA responsável pela baixa digestão da parede celular no rúmen (VAN SOEST, 1994).

Quanto aos parâmetros de France, os quais descrevem numericamente a cinética de fermentação ruminal, nota-se que o potencial máximo de produção de gases (A) da ração contendo 0\% de FMBI possui valor superior aos demais, o que pode levar à dedução de um menor tempo de colonização que as demais rações (Tabela 6). Isso, provavelmente em virtude da ração com $0 \%$ de FMBI ser constituída basicamente de milho e soja, alimentos que possuem alta fermentabilidade e de fácil colonização por microrganismos ruminais (CABRAL et al., 2002).

O parâmetro tempo de colonização (horas) apresentou resultado numericamente inverso, no entanto biologicamente convergente, pois quanto menor o valor de tempo de colonização, menos tempo é necessário para o inicio da fermentação do substrato, o que torna a ração mais fermentável. Os maiores valores da degradabilidade efetiva estão de acordo com os dados de potencial máximo de produção de gases resultando nas mesmas implicações.

As equações demonstram a produção cumulativa de gases da farinha do mesocarpo do babaçu nos diferentes níveis de inclusão (Tabela 7). Observou-se que à medida que aumenta os níveis de inclusão da FMBI na ração, reduz proporcionalmente a produção de gases por tempo de incubação, indicando que a FMBI é um alimento de baixo valor nutricional. No entanto seu uso é economicamente viável por apresentar baixo custo, em relação a alimentos mais caros, como por exemplo, o milho. No entanto, isso pode não ser verdade para animais com maior exigência nutricional, quando a redução do custo pode vir acompanhada por uma diminuição significativa da produtividade.

A inclusão da FMB II no concentrado proporcionou o aumento dos valores de lignina na composição bromatológica das rações (Tabela 8).

Quanto aos parâmetros de France, os quais descrevem numericamente a cinética de fermentação ruminal, nota-se que o potencial máximo de produção de gases (A) da ração contendo $0 \%$ de inclusão de FMBII $^{\circledR}$ possui valor superior aos demais, o que pode

Tabela 5 - Valores de composição bromatológica das rações com níveis crescentes de farinha do mesocarpo do babaçu I ${ }^{\circledR}$ (FMBI)

\begin{tabular}{|c|c|c|c|c|}
\hline \multirow{2}{*}{ Parâmetros bromatológicos } & \multicolumn{4}{|c|}{ Níveis de inclusão de FMB I das rações (\%) } \\
\hline & 0,0 & 7,5 & 15,0 & 22,5 \\
\hline Matéria seca (MS) (\% da Matéria Natural) & 90,67 & 90,43 & 90,20 & 89,97 \\
\hline Proteína bruta (\% da MS) & 12,36 & 12,37 & 12,37 & 12,39 \\
\hline Extrato etéreo (\% da MS) & 2,54 & 2,44 & 2,33 & 2,23 \\
\hline Fibra em detergente neutro ( $\%$ da MS) & 43,58 & 43,52 & 43,49 & 43,45 \\
\hline Fibra em detergente ácido (\% da MS) & 23,34 & 24,16 & 24,99 & 25,82 \\
\hline Lignina (\% da MS) & 3,27 & 4,55 & 5,83 & 7,11 \\
\hline Nutrientes digestíveis totais (\% da MS) & 59,66 & 59,60 & 59,54 & 59,50 \\
\hline
\end{tabular}


Tabela 6 - Parâmetros da cinética de fermentação ruminal in vitro de rações com inclusão farinha do mesocarpo do babaçu I ${ }^{\circledR}$ (FMBI), utilizando o modelo de France e degradabilidade efetiva

\begin{tabular}{lcccc}
\hline \multirow{2}{*}{ Parâmetros de France } & \multicolumn{4}{c}{ Níveis de inclusão de FMBI das rações $(\%)$} \\
\cline { 2 - 4 } & 0,0 & 7,5 & 15,0 & 22,5 \\
\hline $\mathrm{A}^{*}$ & 265,91 & 258,10 & 253,99 & 250,66 \\
$\mathrm{~T}^{*}$ (horas:minutos) & $1: 50$ & $2: 40$ & $2: 51$ & $2: 20$ \\
$\mu^{*}$ & 0,05993 & 0,05978 & 0,05631 & 0,05524 \\
DE** (5\%) & 70,99 & 66,57 & 64,29 & 65,98 \\
DE (6\%) & 69,00 & 64,21 & 61,83 & 63,85 \\
DE (7\%) & 67,05 & 61,92 & 59,46 & 61,76 \\
DE (8\%) & 65,16 & 59,70 & 57,17 & 59,74 \\
\hline
\end{tabular}

A - Total de gases $(\mathrm{mL}) ; \mathrm{T}$ - Tempo de colonização (horas); $\mu$ - Taxa de degradação fracional $\left(\mathrm{h}^{-1}\right) ;$ * Parâmetros estimados pelo modelo de France et al. (1993); ** Degradabilidade efetiva da matéria seca

Tabela 7 - Equações da produção acumulativa de gases (PCG), em mL.g ${ }^{-1}$ de MS de rações com inclusão de farinha do mesocarpo do babaçu I ${ }^{\circledast}$

\begin{tabular}{lcc}
\hline \multicolumn{1}{c}{ Rações* } & Equações (Modelo de France) & $\mathrm{R}^{2}(\%)$ \\
\hline 0,0 & $\mathrm{Y}=265,9173 \times\left\{1-\exp ^{[-(0,0743) \times(\mathrm{t}-1,8699)-(-0,1069) \times(\sqrt{ } \mathrm{t}-\sqrt{1,8699)]}\} \text { a A }}\right.$ & 98,80 \\
7,5 & $\mathrm{Y}=258,1030 \times\left\{1-\exp ^{[-(0,0771) \times(\mathrm{t}-2,6481)-(-0,1333) \times(\sqrt{ } \mathrm{t}-2,6481)]}\right\} \mathrm{b} \mathrm{B}$ & 96,70 \\
15,0 & $\mathrm{Y}=253,9985 \times\left\{1-\exp ^{[-(0,0728) \times(\mathrm{t}-2,9275)-(-0,1297) \times(\sqrt{ } \mathrm{t}-\sqrt{2,9275)]}\} \mathrm{b} \mathrm{B}}\right.$ & 98,20 \\
22,5 & $\mathrm{Y}=250,6609 \times\left\{1-\exp ^{[-(0.0697) \times(\mathrm{t}-2,3206)-(0,1119) \times(\sqrt{ } \mathrm{t}-\sqrt{ } 2,3206)]}\right\} \mathrm{c} \mathrm{C}$ & 97,20 \\
\hline
\end{tabular}

*Níveis de inclusão de FMBI das rações (\%); Equações acompanhadas por letras minúsculas, iguais na mesma coluna, são paralelas pelo teste de paralelismo de curvas a 5\%. Equações acompanhadas por letras maiúsculas, iguais na mesma coluna, são idênticas pelo teste de identidade de curvas a 5\% (REGAZZI e SILVA, 2004)

Tabela 8 - Composição bromatológica percentual de rações com inclusão farinha do mesocarpo do babaçu II ${ }^{\circledR}$ (FMBII)

\begin{tabular}{|c|c|c|c|c|}
\hline \multirow{2}{*}{ Parâmetros bromatológicos } & \multicolumn{4}{|c|}{ Níveis de inclusão de FMBII das rações (\%) } \\
\hline & 0,0 & 7,5 & 15,5 & 22,5 \\
\hline Matéria seca (MS) (\% da Matéria Natural) & 90,67 & 90,43 & 90,24 & 90,02 \\
\hline Proteína bruta (\% da MS) & 12,37 & 12,38 & 12,40 & 12,43 \\
\hline Extrato etéreo (\% da MS) & 2,54 & 2,35 & 2,16 & 1,97 \\
\hline Fibra em detergente neutro ( $\%$ da MS) & 43,56 & 42,92 & 42,27 & 41,63 \\
\hline Fibra em detergente ácido (\% da MS) & 23,33 & 23,73 & 24,12 & 24,52 \\
\hline Lignina (\% da MS) & 3,27 & 3,91 & 4,55 & 5,18 \\
\hline Nutrientes digestíveis totais (\% da MS) & 59,67 & 59,94 & 60,23 & 60,52 \\
\hline
\end{tabular}

levar a dedução de menor fermentação das demais rações (Tabela 9).

O parâmetro tempo de colonização (horas) apresentou resultado inverso, no entanto biologicamente convergente, pois quanto menor o valor de tempo de colonização, mais tempo é necessário para o início da fermentação do substrato, o que torna a ração menos fermentável. Todavia, a inclusão FMBII na ração aumenta o tempo de colonização e esse aumento está diretamente relacionado com aumento do valor de FDN.

Velásquez et al. (2009) observaram maior tempo para colonização dos substratos de gramíneas com valores 
Tabela 9 - Parâmetros da cinética de fermentação ruminal in vitro de rações com inclusão farinha do mesocarpo do babaçu II ${ }^{\circledR}$ (FMBII), utilizando o modelo de France e degradabilidade efetiva

\begin{tabular}{lcccc}
\hline \multirow{2}{*}{ Parâmetros de France } & \multicolumn{4}{c}{ Níveis de inclusão de FMBII das rações (\%) } \\
\cline { 2 - 5 } & 0,0 & 7,5 & 15,0 & 22,5 \\
\hline $\mathrm{A}^{*}$ & 217,0964 & 199,2681 & 194,4534 & 195,7873 \\
$\mathrm{~T}^{*}$ (Horas:minutos) & $1: 01$ & $2: 04$ & $2: 10$ & $2: 35$ \\
$\mu^{*}$ & 0,038929 & 0,051086 & 0,036922 & 0,040828 \\
$\mathrm{DE} * *(2 \%)$ & 60,6715 & 59,1412 & 57,9138 & 61,5358 \\
$\mathrm{DE}(3 \%)$ & 60,0632 & 58,5721 & 57,3151 & 60,9118 \\
$\mathrm{DE}(4 \%)$ & 59,4553 & 58,0032 & 56,7172 & 60,2885 \\
$\mathrm{DE}(5 \%)$ & 58,8479 & 57,4344 & 56,1200 & 59,6658 \\
\hline
\end{tabular}

A - Total de gases (mL); T - Tempo de colonização (horas); $\mu$ - Taxa de degradação fracional ( $\left.\mathrm{h}^{-1}\right) ;{ }^{*}$ Parâmetros estimados pelo modelo de France et al. (1993); ** Degradabilidade efetiva da matéria seca

altos de FDN. Muniz et al. (2011) relataram que os teores menores de FDN, resultam em maior produção de gás, devido a maior proporção de conteúdo celular, e, com isso, menor tempo de colonização pelos microrganismos em palma forrageira. Os maiores valores da degradabilidade efetiva estão de acordo com os dados de potencial máximo de produção de gases resultando nas mesmas implicações.

As equações advindas da análise de regressão dos dados de cinética de fermentação ruminal das rações com inclusão de FMBII ${ }^{\circledR}$ (Tabela 10) apresentaramse distintas, provavelmente em função das diferenças bromatológicas da farinha de mesocarpo de babaçu.

Observa-se que à medida que aumenta a proporção de FMBII $^{\circledR}$ na ração, reduz-se a produção de gases por tempo de incubação, indicando que a FMBII $^{\circledR}$ é um alimento com menor valor nutritivo que os alimentos que ele substitui na ração, principalmente o milho (de baixo valor nutricional). No entanto seu uso é economicamente viável por apresentar baixo custo, em relação a alimentos mais caros, como por exemplo, o milho. A inclusão crescente da FMBII ${ }^{\circledR}$ na ração elevouse os teores de lignina, sendo um fator importante para a redução da degradabilidade da ração.

O volume de gases produzidos sofre interferência da relação propionato/acetato, sendo que a maior produção de acetato favorece a produção de gases, o aumento da produção do propionato, ocorre um abaixamento do $\mathrm{pH}$, diminuindo a produção de gases (AZARFAR et al., 2009). As rações experimentais geralmente, em sistemas "in vitro", não ocasionam um abaixamento excessivo do $\mathrm{pH}$ ruminal, devido aos tamponantes da solução de saliva artificial, no entanto a relação propionato/acetato pode ser alterada em rações experimentais como essas com níveis crescentes de inclusão das farinhas com mesocarpo de babaçu que apresentam uma elevada fração fibrosa não digestível, influenciando a fermentação e, consequentemente, a produções de gases.

Tabela 10 - Equações da produção acumulativa de gases, em mL.g-1 de MS das rações com diferentes níveis de inclusão de farinha do mesocarpo do babaçu II ${ }^{\circledR}$

\begin{tabular}{|c|c|c|}
\hline Rações* & Equações (Modelo de France) & $\mathrm{R}^{2}(\%)$ \\
\hline 0,0 & $Y=217,0964 \times\left\{1-\exp ^{[-0,0577(t-0,0376)-(-0,1544) \times(\sqrt{ } t-\sqrt{ } 0,0376)]}\right\}$ a $A$ & 98,20 \\
\hline 7,5 & $Y=199,2681 \times\left\{1-\exp ^{[-0,0543(t-0,0118)-(-0,1492) \times(\sqrt{ } t-\sqrt{ } 0,0118)]}\right\}$ a B & 98,40 \\
\hline 15,0 & $Y=194,4534 \times\left\{1-\exp ^{[-0,0516(t-0,0669)-(-0,1465) \times(\sqrt{ } t-\sqrt{ } 0,0669)]}\right\}$ a $C$ & 98,00 \\
\hline 22,7 & $Y=195,7873 \times\left\{1-\exp ^{[-0,0522(t-0,0525)-(-0,1453) \times(\sqrt{ } t-\sqrt{ } 0,0525)]}\right\}$ a $C$ & 92,70 \\
\hline
\end{tabular}

*Níveis de inclusão de FMBII das rações (\%); Equações acompanhadas por letras minúsculas, iguais na mesma coluna, são paralelas pelo teste de paralelismo de curva a 5\%. Equações acompanhadas por letras maiúsculas, iguais na mesma coluna, são idênticas pelo teste de identidade de curvas a 5\% (REGAZZI E SILVA, 2004) 


\section{CONCLUSÃO}

A farinha do mesocarpo do babaçu $\mathrm{I}^{\circledR}$ e $\mathrm{II}^{\circledR}$, quando adicionada ao concentrado em níveis iguais ou acima de 7,5\%, resulta em redução da fermentação ruminal de rações. Embora não apresente alto valor nutritivo, esses alimentos apresentam potencial quando usados como constituintes nas rações para ruminantes de menor exigência nutricional.

\section{REFERÊNCIAS}

ABDALLA, A. L. et al. Utilização de subprodutos da indústria de biodiesel na alimentação de ruminantes. Revista Brasileira de Zootecnia, v. 37, p. 260-268, 2008. Número Especial.

AZARFAR, A. et al. In vitro gas production profiles and fermentation end-products in processed barley, maize and milo. Journal of the Science of Food and Agriculture, v. 89, n. 10, p. 1697-1708, 2009.

ASSOCIATION OF OFFICIAL ANALYTICAL CHEMISTS. Official methods of analysis of AOAC international. 17. ed. Washington, 2002.

CABRAL, L. da S. et al. Cinética ruminal das frações de carboidratos, produção de gás, digestibilidade in vitro da matéria seca e NDT estimado da silagem de milho com diferentes proporções de grãos. Revista Brasileira de Zootecnia, v. 31, n. 6, p. 2332-2339, 2002.

EMPRESA BRASILEIRA DE PESQUISA AGROPECUÁRIA. Babaçu - Programa Nacional de Pesquisa, Departamento de Orientação e Apoio à Programação de Pesquisa. Brasília: EMBRAPA, 1984.

FRANCE, J. et al. A model to interpret gas accumulation profiles associated with in vitro degradation of ruminant feeds. Journal Theoretical Biology, v. 163, n. 1, p. 99-111, 1993.

GUIMARÃES JÚNIOR, R. et al. Cinética de fermentação ruminal de silagens de milheto. Arquivo Brasileiro de Medicina Veterinária e Zootecnia, v. 60, n. 5, p. 1174-1180, 2008.

KRISHNAMOORTHY, U. et al. The in vitro gas production technique: Limitations and opportunities. Animal Feed Science and Technology, v. 123/124, n. 1, p. 1-7, 2005.

MENDES, A. R. et al. Consumo e digestibilidade total e parcial de dietas utilizando farelo de girassol e três fontes de energia em novilhos. Revista Brasileira de Zootecnia, v. 34, n. 2, p. 679-691, 2005.
MENKE, K. H. et al. The estimation of the digestibility and metabolizable energy content of ruminant feedingstuffs from the gas production when they are incubated with rumen liquor in vitro. Journal of Agricultural Science, v. 93, n. 1, p. 217-222, 1979.

MERTENS, D. R. Creating a system for meeting the fiber requirements of dairy cows. Journal Dairy Science, v. 80, n. 7 , p. 1463-1481, 1997.

MUNIZ, E. B. et al. Cinética de degradação ruminal de carboidratos de volumosos secos e aquosos: técnica de produção de gases. Semina: Ciência Agrária, v. 32, n. 3, p. 1191-1200, 2011.

NEIVA JÚNIOR, A. P. et al. Efeito de diferentes aditivos sobre os teores de proteína bruta, extrato etéreo e digestibilidade da silagem de maracujá. Ciência e Agrotecnologia, v. 31, n. 3, p. 871-875, 2007.

REGAZZI, A. J.; SILVA, C. H. O. Teste para verificar a igualdade de parâmetros e a identidade de modelos de regressão não-linear. Revista de Matemática e Estatística, v. 22, n. 2, p. 33-45, 2004.

RYMER C. et al. In vitro cumulative gas production techniques: History, methodological considerations and challenges. Animal Feed Science and Technology, v. 123124, n. 1, p. 9-30, 2005.

SANTOS, R. A. et al. Estimativa da degradabilidade ruminal de alimentos utilizando a técnica de produção de gás em bovinos, ovinos e caprinos. Revista Ciência e Agrotecnologia, v. 27, n. 3, p. 689-695, 2003.

SOLER, M. P. et al. Tecnologia de quebra do coco babaçu (Orbignya speciosa). Ciência e Tecnologia de Alimentos, v. 27, n. 4, p. 717-722, 2007.

SALLAM, S. M. A. et al. Use of an in vitro rumen gas production technique to evaluate some ruminants feedstuffs. Journal Applied Science Research, v. 3, n. 1, p. 34-41, 2007.

TOBASA BIOINDUSTRIAL DE BABAÇU S.A. Nossos Produtos. Disponível em: <http://www.tobasa.com.br/ nossos_produtos.html>. Acesso em: 01 dez. 2011.

VELÁSQUEZ, P. A. T. et al. Cinética da fermentação e taxas de degradação de forrageiras tropicais em diferentes idades de corte estimadas pela técnica de produção de gases in vitro. Revista Brasileira de Zootecnia, v. 38, n. 9, p. 1695-1705, 2009.

VAN SOEST, P. J. Nutritional ecology of the ruminant. 2. ed. Ithaca: Cornell University Press, 1994. 476 p. 